\title{
Retrobulbar ocular blood flow changes measured by colour Doppler imaging after intra-arterial chemotherapy in retinoblastoma
}

\author{
Kang Xue, ${ }^{1}$ Ailin Liu, ${ }^{1}$ Ren Hui, ${ }^{1}$ Jing Zhang, ${ }^{2}$ Jiang Qian ${ }^{1}$
}

${ }^{1}$ Department of Ophthalmology and Shanghai Key Laboratory of Visual Impairment and Restoration, Eye, Ear, Nose, and Throat Hospital of Fudan University, Shanghai, China ${ }^{2}$ Guangzhou Women and Children's Medical Center, Guangzhou, China

\section{Correspondence to}

Dr Jiang Qian, Department of Ophthalmology, Eye, Ear, Nose and Throat Hospital of Fudan University, Shanghai 200031, China;

qianjiang58@hotmail.com

$\mathrm{KX}$ and $\mathrm{AL}$ contributed equally.

Received 13 December 2016 Revised 10 January 2017 Accepted 27 January 2017 Published Online First 22 February 2017
CrossMark

To cite: Xue K, Liu A, Hui R, et al. Br J Ophthalmol 2017;101:1419-1422.

\section{ABSTRACT}

Purpose To evaluate the effects of intra-arterial chemotherapy on retrobulbar blood flow parameters in patients with retinoblastoma.

Methods 20 eyes of 10 patients with unilateral retinoblastoma that were treated with intra-arterial chemotherapy were evaluated using colour Doppler imaging. The peak systolic and end-diastolic velocities of the ophthalmic, central retinal and posterior ciliary arteries were determined. The pulsatility and resistance indices were calculated automatically. The treated eye was compared with the untreated (control) eye and with itself before and after intra-arterial chemotherapy.

Results When comparing the retinoblastomacontaining eyes with the contralateral normal eyes, the peak systolic and end-diastolic velocities of the central retinal artery were significantly higher in the tumorous eyes than in the normal eyes before intra-arterial chemotherapy. Moreover, the peak systolic and enddiastolic velocities in the posterior ciliary and central retinal arteries were significantly decreased after intraarterial chemotherapy in the tumorous eyes $(p<0.05)$. There were no statistically significant differences in the other parameters.

Conclusions Our results suggest that intra-arterial chemotherapy has a measurable effect on the retrobulbar blood flow, which can cause a decrease in the peak systolic and end-diastolic velocities in the posterior ciliary and central retinal arteries.

\section{INTRODUCTION}

Retinoblastoma is the most common paediatric intraocular malignancy, occurring in approximately 1 in 15 000-20 000 live births. ${ }^{1}$ For over a decade, treatment has been focused on globe-preserving techniques, including systemic chemotherapy and a combination of focal consolidative therapy. ${ }^{2-4}$ Recently, superselective intra-arterial chemotherapy (IAC) for the treatment of retinoblastoma has gained popularity and has begun to be used as a first-line treatment. $^{5-7}$ However, vaso-occlusive disease has been described following superselective intraophthalmic arterial chemotherapy as a potentially sight-threatening complication, ${ }^{8-10}$ and ophthalmologists should be aware of such vascular complications. Colour Doppler imaging (CDI) is one of the most important diagnostic methods for detecting haemodynamic alterations in orbital vessels. ${ }^{11} 12$ However, there are limited reports in the literature about CDI changes of these vessels after IAC. In this study, we prospectively evaluated the effects of intra-arterial melphalan on the retrobulbar blood flow parameters in patients with retinoblastoma via CDI to determine a correlation between the clinical findings and IAC.

\section{METHODS}

\section{Ethics statement}

This research was conducted in strict accordance with the tenets of the Declaration of Helsinki. Written informed consent was obtained from the parents, caretakers or guardians on behalf of all of the children, and placed in the patient record. Consent was given for the use of this established, previously published off-label treatment for chemotherapy.

\section{Patients}

The medical records of 10 patients diagnosed with unilateral retinoblastoma and treated with IAC at the Department of Ophthalmology, Eye and Ear, Nose and Throat Hospital of Fudan University in Shanghai, China, from January 2014 to December 2015, were reviewed for this study. The exclusion criteria were eyes with bilateral tumours and painful glaucoma, buphthalmos, anterior chamber seeding, diffuse infiltrating retinoblastoma or phthisis bulbi, which were indications for primary enucleation. ${ }^{13}$

\section{Surgical technique}

IAC was performed by a skilled radiologist (JZ) specialised in the intervention procedure. In order to reduce potential thrombotic complications, intravenous heparin $(70 \mathrm{U} / \mathrm{kg})$ was used during the procedure. A femoral arterial puncture and the insertion of a Hunter catheter passing through the aorta, common carotid artery and internal carotid artery were conducted, and a Marathon microcatheter was positioned at the ostium of the ophthalmic artery (OA). The melphalan dose was 3, 5 or $7.5 \mathrm{mg}$, increasing with the patient's age and tumour size. The carboplatin dose was $20 \mathrm{mg}$, while each chemotherapy dose was diluted in $30 \mathrm{~mL}$ of saline and administered in a pulsatile fashion over $30 \mathrm{~min}^{7}$

\section{Colour Doppler imaging measurements}

The sonographer (AL) performed the orbital CDI studies preoperatively and after the IAC cycles. The blood flow in the retrobulbar vessels was assessed by a Philips high definition imaging 5000 CDI system (Philips Ultrasound, Bothell, Washington, USA) with a $7.5 \mathrm{MHz}$ linear probe, used as described by Founti ${ }^{14}$ and colleagues with a paraocular approach, while the patients were in the supine position. All of the patients were examined under oral chloral hydrate sedation $(0.5 \mathrm{~mL} / \mathrm{kg})$. 
The samples of the pulsed Doppler signal from within a $0.2 \times 0.2 \mathrm{~mm}$ sample area were analysed to calculate the blood velocities in the retrobulbar vasculature. The CDI measurements were taken in the OA, central retinal artery (CRA) and posterior ciliary artery (PCA), and the peak systolic velocity (PSV) and end-diastolic velocity (EDV) were determined in each vessel. The IAC procedures do not change the anatomical location of retrobulbar ocular vessels. The CRA should always be measured within the centre of the optic nerve shadow, 1-2 mm behind the surface of the optic nerve. To measure the OA blood flow velocities, the Doppler sample gate was set $10-15 \mathrm{~mm}$ posterior to the globe. Appropriate angle correction was used for the OA measurement. Angle correction was not applied in CRA or PCA because of the coincidence of the axis of arteries with the sound beam. We applied the same parameter and angle before and after IAC to ensure the measurements were comparable. The pulsatility and resistance indices were calculated automatically by the scanner, after measuring the systolic and diastolic blood flow velocities of each vessel, and the appropriate angle correction was used for the vessels measured. All of the parameters were recorded three times during the same session, and the mean values were used in the statistical analyses.

\section{Statistical analysis}

The treated eye was compared with the untreated (control) eye in the same patient, and the comparisons were made preoperatively and after the IAC cycles. The data are presented as the mean value with the SD. The comparisons of the preoperative and postoperative values and the differences between the treated and control eyes were performed using the paired t-test or Wilcoxon signed-rank test, depending on the data distribution. A value of $\mathrm{p}<0.05$ was considered to be statistically significant.

\section{RESULTS}

Ten eyes of 10 patients who underwent a total of 34 IAC treatments for unilateral retinoblastoma were analysed. Six of the patients were male, and four were female, and the mean age at diagnosis was $17.3 \pm 4.3$ months old. In the treated eyes, the International Classification of Retinoblastoma groups were D $(n=7)$ and E $(n=3)$. The IAC was delivered as the primary therapy in seven eyes and as a salvage therapy in three eyes. The demographic characteristics and tumour features are listed in table 1. None of the patients in this series had a stroke, seizure, neurological impairment, limb ischaemia, secondary leukaemia or metastasis, and none died. No IAC-related serious catheterisation complications (carotid artery spasm, OA thrombosis, vasospasm or artery injury) were present in any of the 34 procedures. Catheterisations were successful in all procedures. The mean number of IAC treatments was 3.4 \pm 1.5 (median, 3; range, 2-5), and the mean interval from the last IAC to the CDI measurements was $6.8 \pm 1.4$ weeks (median, 6 weeks; range, 39 weeks). The mean total dose of melphalan received per patient was $15.8 \pm 8.3 \mathrm{mg}$ (median, $15 \mathrm{mg}$; range, 5-30.5 mg). The clinical features and outcomes are listed in table 2.

The mean retrobulbar blood flow values of the OA, PCA and CRA before and after IAC are shown in table 3. After comparing the retinoblastoma-containing eyes with the contralateral normal eyes, the PSV and EDV of the CRA were significantly higher in the tumorous eyes than in the normal eyes before the IAC treatment. The PSV and EDV in the PCA and CRA were significantly decreased after IAC in the tumorous eyes $(p<0.05)$ (Patient 5, figures 1 and 2). When compared with the normal controls after IAC, the decrease in the EDV in the CRA was
Table 1 Unilateral retinoblastoma treated with IAC in 10 patients: demographics and tumour features

\begin{tabular}{llllllll}
\hline Patient & $\begin{array}{l}\text { Age } \\
\text { (months) }\end{array}$ & Sex & $\begin{array}{l}\text { Affected } \\
\text { eye }\end{array}$ & Treatment & ICRB & SRS & VS \\
\hline 1 & 20 & F & OS & Primary & E & + & + \\
2 & 18 & M & OD & Primary & D & - & + \\
3 & 22 & M & OD & Primary & E & + & + \\
4 & 16 & M & OS & Primary & D & + & + \\
5 & 11 & M & OS & Primary & D & + & + \\
6 & 20 & M & OS & Salvage & D & - & + \\
7 & 14 & F & OS & Primary & D & + & + \\
8 & 24 & F & OD & Primary & E & + & + \\
9 & 9 & F & OD & Salvage & D & - & + \\
10 & 19 & M & OD & Salvage & D & - & + \\
\hline
\end{tabular}

F, female; IAC, intra-arterial chemotherapy; ICRB, International Classification of Retinoblastoma; M, male; OD, right eye; OS, left eye; SRS, subretinal seeds; VS, vitreous seeds.

Table 2 Unilateral retinoblastoma treated with intra-arterial chemotherapy (IAC) in 10 patients: treatment and outcomes

\begin{tabular}{|c|c|c|c|c|c|}
\hline Patient & $\begin{array}{l}\text { Number } \\
\text { of IAC } \\
\text { cycles }\end{array}$ & $\begin{array}{l}\text { Tumour } \\
\text { control } \\
\text { achieved }\end{array}$ & $\begin{array}{l}\text { Catheterisation } \\
\text { complications }\end{array}$ & $\begin{array}{l}\text { Fundus } \\
\text { evidence of } \\
\text { choroidal } \\
\text { atrophy }\end{array}$ & $\begin{array}{l}\text { Fundus } \\
\text { evidence } \\
\text { of vascular } \\
\text { stenosis or } \\
\text { occlusion }\end{array}$ \\
\hline 1 & 3 & Partial & No & - & + \\
\hline 2 & 3 & Total & No & + & + \\
\hline 3 & 5 & Partial & No & + & - \\
\hline 4 & 2 & Total & No & - & - \\
\hline 5 & 3 & Total & No & + & + \\
\hline 6 & 4 & Partial & No & - & - \\
\hline 7 & 3 & No & No & - & - \\
\hline 8 & 2 & Total & No & + & + \\
\hline 9 & 3 & Total & No & - & - \\
\hline 10 & 6 & Partial & No & + & - \\
\hline
\end{tabular}

significant $(p<0.05)$; however, the decrease in the PSV in the CRA or PSV and EDV in the PCA did not reach significance. There were no statistically significant differences in the other parameters.

\section{DISCUSSION}

Although the intra-arterial infusion of melphalan has shown promising results, ophthalmologists have noted several side effects, among which vascular toxicity has been described as a potentially sight-threatening complication. Post treatment, Munier et $a l^{9}$ found that 3 of 13 (23\%) patients had choroidal ischaemia or retinal arteriolar emboli. Shields et $a l^{10}$ found that 6 of 17 eyes (35\%) had choroidal vasculopathy or retinal artery occlusion. The CRA supplies blood to the retina, while the PCA supplies blood to the optic nerve head and the choroidal layer. Therefore, the measurements of the blood flow velocities in the PCA and CRA may be good indicators of the capillary blood flow of the optic nerve head, retina and choroidal layer. Since it is impossible to determine the diameter of the orbital vessels in vivo, the blood flow velocity can be estimated by spectral Doppler analysis. CDI has been used with success in the assessment of retrobulbar vessel haemodynamics in healthy eyes and eyes with retinal disease, and in detecting haemodynamic 
Table 3 Haemodynamic Doppler parameters in the OA, PCA and CRA in 10 eyes in patients with retinoblastoma before and after IAC

\begin{tabular}{|c|c|c|c|c|c|c|c|c|}
\hline \multirow[b]{2}{*}{ Parameter } & \multicolumn{3}{|c|}{ Treated eye (treated) } & \multicolumn{3}{|c|}{ Untreated eye (control) } & \multirow{2}{*}{$\begin{array}{l}\text { T vs C pre-IAC } \\
\text { p Value }\end{array}$} & \multirow{2}{*}{$\begin{array}{l}\text { T vs } C \text { post-IAC } \\
\text { p Value }\end{array}$} \\
\hline & Pre-IAC & Post-IAC & $\mathrm{p}$ Value & Pre-IAC & Post-IAC & $\mathrm{p}$ Value & & \\
\hline \multicolumn{9}{|c|}{ Central retinal artery } \\
\hline PSV (cm/s) & $13.88 \pm 3.02$ & $7.55 \pm 2.21$ & 0.0000 & $9.64 \pm 2.16$ & $9.42 \pm 2.30$ & 0.8208 & 0.0011 & 0.0655 \\
\hline $\mathrm{EDV}(\mathrm{cm} / \mathrm{s})$ & $4.69 \pm 1.28$ & $1.80 \pm 0.71$ & 0.0000 & $2.68 \pm 1.08$ & $2.69 \pm 0.82$ & 0.9816 & 0.0013 & 0.0184 \\
\hline $\mathrm{RI}$ & $0.75 \pm 0.08$ & $0.68 \pm 0.07$ & 0.0717 & $0.70 \pm 0.11$ & $0.68 \pm 0.12$ & 0.7580 & 0.2627 & 0.9818 \\
\hline $\mathrm{PI}$ & $1.12 \pm 0.23$ & $1.11 \pm 0.22$ & 0.9285 & $1.10 \pm 0.22$ & $1.13 \pm 0.23$ & 0.7809 & 0.8972 & 0.8111 \\
\hline \multicolumn{9}{|l|}{ PCA } \\
\hline PSV $(\mathrm{cm} / \mathrm{s})$ & $10.73 \pm 1.96$ & $8.29 \pm 2.03$ & 0.0137 & $9.77 \pm 2.46$ & $9.64 \pm 2.55$ & 0.9089 & 0.3472 & 0.2070 \\
\hline $\mathrm{EDV}(\mathrm{cm} / \mathrm{s})$ & $3.46 \pm 1.04$ & $2.22 \pm 1.01$ & 0.0148 & $3.11 \pm 1.19$ & $3.08 \pm 1.31$ & 0.9578 & 0.4928 & 0.1177 \\
\hline $\mathrm{RI}$ & $0.67 \pm 0.12$ & $0.72 \pm 0.14$ & 0.4220 & $0.61 \pm 0.09$ & $0.68 \pm 0.11$ & 0.9780 & 0.7500 & 0.5499 \\
\hline $\mathrm{PI}$ & $1.03 \pm 0.26$ & $1.16 \pm 0.36$ & 0.3628 & $1.05 \pm 0.19$ & $1.06 \pm 0.23$ & 0.9798 & 0.7980 & 0.4651 \\
\hline \multicolumn{9}{|c|}{ Ophthalmic artery } \\
\hline PSV (cm/s) & $24.25 \pm 5.98$ & $22.61 \pm 5.58$ & 0.5342 & $22.26 \pm 5.04$ & $21.84 \pm 4.07$ & 0.8399 & 0.4318 & 0.7286 \\
\hline $\operatorname{EDV}(\mathrm{cm} / \mathrm{s})$ & $6.98 \pm 1.63$ & $7.11 \pm 1.43$ & 0.8518 & $6.46 \pm 1.22$ & $6.59 \pm 1.41$ & 0.8278 & 0.4298 & 0.4235 \\
\hline $\mathrm{RI}$ & $0.75 \pm 0.08$ & $0.72 \pm 0.06$ & 0.3463 & $0.72 \pm 0.08$ & $0.71 \pm 0.09$ & 0.7044 & 0.4411 & 0.7421 \\
\hline PI & $1.72 \pm 0.47$ & $1.65 \pm 0.44$ & 0.7367 & $1.68 \pm 0.45$ & $1.64 \pm 0.38$ & 0.8284 & 0.8523 & 0.9574 \\
\hline
\end{tabular}

Bold indicates statistically significant differences $(p<0.05)$.

CRA, central retinal artery; EDV, end-diastolic velocity; IAC, intra-arterial chemotherapy; OA, ophthalmic artery; PCA, posterior ciliary artery; PI, pulsatility; PSV, peak systolic velocity; RI, resistance.

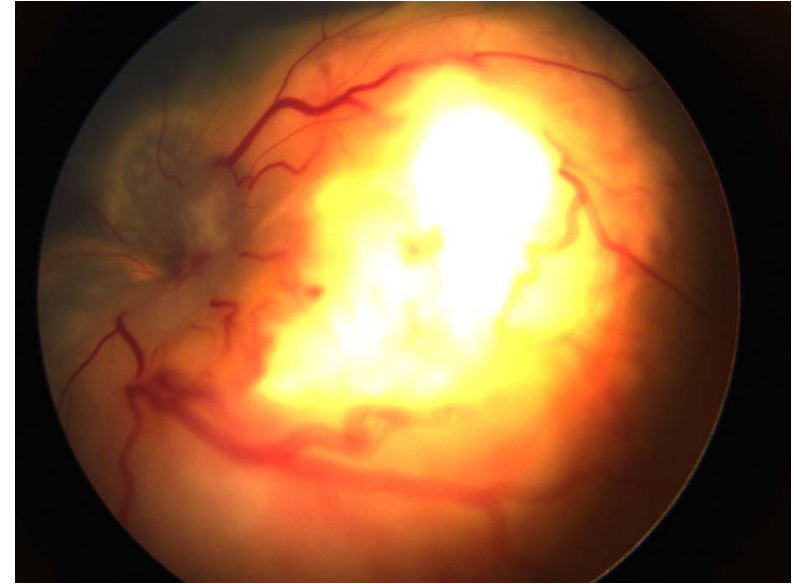

Figure 1 Patient 5. RetCam III previously showed large tumour (figure 1).

alterations in the orbital vessels. ${ }^{15} 16$ These individual vessels can be investigated, and the Doppler frequency shifts are received from a specific sample volume.

Steinle et al undertook parallel in vitro and in vivo lines of research and indicated that IAC may trigger vascular toxicity through endothelial cell inflammation and leucostasis. The vascular toxicity may be related to the $\mathrm{pH}$, the pulsatile delivery or the chemotherapeutic drugs used. ${ }^{17}$ In a non-human primate model, the retinal arterioles revealed smudging of the endothelial cells and their basement membranes with terminal vessel occlusion. ${ }^{18}$ Maidana et al ${ }^{19}$ have reported thinning of the choroid after IAC, as accessed by optical coherence tomography. In addition, Tse et al presented a case that developed enophthalmos, choroidal ischaemia, iris atrophy and scleritis as delayed toxicities of IAC. They hypothesised that the vascular insult to the PCA led to ischaemia, resulting in iris atrophy and chronic damage to the orbital vasculature, ocular growth impairment and orbital fat atrophy. ${ }^{20}$ In our series, no eyes were enucleated, and we first proposed decreases in the blood flow in the PCA and CRA, but the blood flow remained unchanged in the OA. This proved that IAC may lead to ocular and orbital vessel

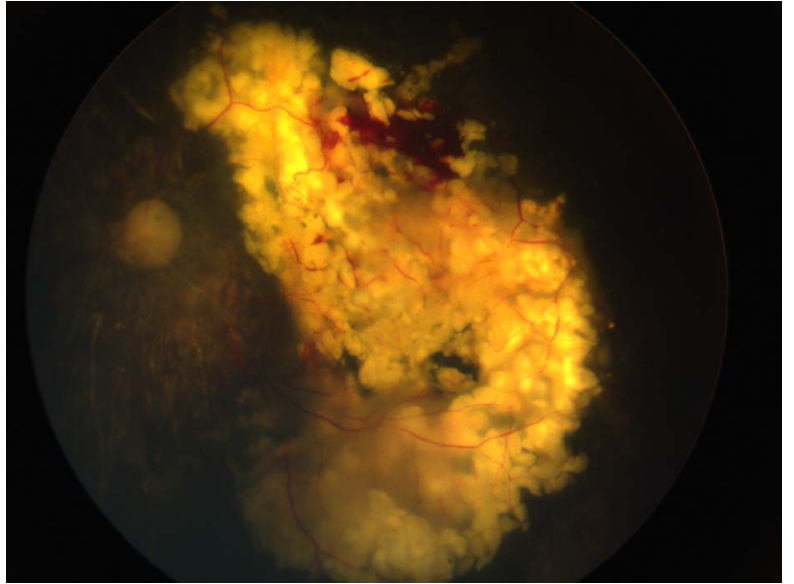

Figure 2 Patient 5. Involved left eye (figure 2) with calcified regression retinoblastoma in the macular region after intra-arterial chemotherapy (IAC). The choroidal atrophy and vascular occlusion were visible. The peak systolic velocity and end-diastolic velocity in the central retinal artery significantly decreased from 22.9 and $7.3 \mathrm{~cm} / \mathrm{s}$ to 3.2 and $3.6 \mathrm{~cm} / \mathrm{s}$ after three IAC cycles in the tumorous eye.

toxicity, which requires close clinical follow-up to assess the long-term consequences.

Before IAC, the PSV and EDV in the CRA and PCA were significantly higher in the tumour-containing eyes than in the contralateral normal eyes, indicating a haemodynamic change in the retrobulbar vessels caused by the presence of a large and malignant tumour. As for the reason, a retinoblastoma is a rapidly growing malignant tumour that requires an additional blood supply, triggering the sequence of events that cause elevations in the PSV and EDV in the CRA and PCA, and consistent with the results of Bonanomi. ${ }^{21}$ The decreases in the blood flow velocities of the retrobulbar circulation were in part due to the regression of the tumour, indicating a response to the therapeutic approach, and partly because of the vascular toxicity from the delivery of higher concentrations of chemotherapy. After IAC, the untreated fellow eyes showed no statistical significance. When compared with the normal controls, the PSV and 
EDV in the PCA and CRA were decreased, but only the decrease of EDV in the CRA reached significance, probably due to the limited number of patients. The OA showed no measurable changes, which suggested that IAC may affect smaller vessels more, rather than bigger vessels like the OA. The IAC technique does not change the blood flow of the OA.

This study was not without certain limitations. First of all, we must mention that the number of participants included was limited and that the follow-up was short. However, the difference in the means was broad, short-term, after IAC. Long-term follow-up should be conducted in the future to show the longterm effects of IAC. For CDI performed under sedation, the shadows in a tumour-containing eye can be challenging; so, we chose either the nasal or temporal PCA to compare the same artery after IAC.

\section{CONCLUSION}

Our results suggest that some significant decreases in the blood flow velocities occur in the retrobulbar circulation in some arteries after IAC. Further clinical studies will clarify the long-term effects of IAC on the retrobulbar blood flow in patients with retinoblastoma.

Correction notice This article has been corrected since it was published Online First. The statement 'This is a case-control study' has been removed from the Abstract as the Editors did not feel this was accurate.

Contributors KX and JQ had full access to all of the data in the study and take responsibility for the integrity of the data and the accuracy of the data analysis. Study concept and design: $\mathrm{KX}, \mathrm{AL}, \mathrm{JZ}, \mathrm{RH}$ and JQ. Acquisition, analysis or interpretation of data: $K X, A L$ and JQ. Drafting of the manuscript: $K X$. Critical revision of the manuscript for important intellectual content: all authors. Statistical analysis: $\mathrm{KX}$ and $\mathrm{AL}$. Obtained funding: $\mathrm{KX}, \mathrm{JQ}$ and $\mathrm{RH}$. Administrative, technical or material support: JZ and JQ.

Funding Funding for this study was provided by the National Health and Family Planning Commission of Shanghai (No. 20144Y0264), the Science and Technology Commission of the Shanghai Municipality (No. 14411961800) and National Natural Science Foundation of China (No.81300805).

Competing interests None declared.

Patient consent Obtained.

Ethics approval The study protocol was approved by the Ethics Committee of the Eye and ENT Hospital of Fudan University.

Provenance and peer review Not commissioned; externally peer reviewed.

\section{REFERENCES}

1 Dimaras H, Kimani K, Dimba EA, et al. Retinoblastoma. Lancet 2012;379:1436-46.
2 Ferris FL, Chew EY. A new era for the treatment of retinoblastoma. Arch Ophthalmol 1996;114:1339-43.

3 Shields CL, Mashayekhi A, Au AK, et al. The international classification of retinoblastoma predicts chemoreduction success. Ophthalmology 2006;113:2276-80.

4 Grossniklaus HE. Retinoblastoma. Fifty years of progress. The LXXI Edward Jackson memorial lecture. Am J Ophthalmol 2014;158:875-91.

5 Suzuki S, Yamane T, Mohri M, et al. Selective ophthalmic arterial injection therapy for intraocular retinoblastoma: the long-term prognosis. Ophthalmology 2011;118:2081-7.

6 Gobin YP, Dunkel IJ, Marr BP, et al. Intra-arterial chemotherapy for the management of retinoblastoma: four-year experience. Arch Ophthalmol 2011;129:732-7.

7 Shields CL, Manjandavida FP, Lally SE, et al. Intra-arterial chemotherapy for retinoblastoma in 70 eyes: outcomes based on the international classification of retinoblastoma. Ophthalmology 2014;121:1453-60.

8 Wilson MW, Jackson JS, Phillips BX, et al. Real-time ophthalmoscopic findings of superselective intraophthalmic artery chemotherapy in a nonhuman primate model. Arch Ophthalmol 2011;129:1458-65.

9 Munier FL, Beck-Popovic M, Balmer A, et al. Occurrence of sectoral choroidal occlusive vasculopathy and retinal arteriolar embolization after superselective ophthalmic artery chemotherapy for advanced intraocular retinoblastoma. Retina (Philadelphia, Pa) 2011;31:566-73.

10 Shields $C L$, Bianciotto CG, Jabbour $P$, et al. Intra-arterial chemotherapy for retinoblastoma: report no. 2, treatment complications. Arch Ophthalmol 2011;129:1407-15.

11 Lieb WE, Cohen SM, Merton DA, et al. Color Doppler imaging of the eye and orbit. Technique and normal vascular anatomy. Arch Ophthalmol 1991;109:527-31.

12 Lieb WE, Shields JA, Cohen SM, et al. Color Doppler imaging in the management of intraocular tumors. Ophthalmology 1990;97:1660-4.

13 Abramson DH, Shields CL, Munier FL, et al. Treatment of retinoblastoma in 2015: agreement and disagreement. JAMA Ophthalmol 2015;133:1341-7.

14 Founti $P$, Harris $A$, Papadopoulou $D$, et al. Agreement among three examiners of color Doppler imaging retrobulbar blood flow velocity measurements. Acta Ophthalmol 2011;89:e631-4.

15 Güven D, Özdemir H, Hasanreisoglu B. Hemodynamic alterations in diabetic retinopathy. Ophthalmology 1996;103:1243-9.

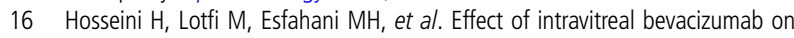
retrobulbar blood flow in injected and uninjected fellow eyes of patients with neovascular age-related macular degeneration. Retina (Philadelphia, Pa) 2012;32:967-71.

17 Steinle JJ, Zhang Q, Thompson KE, et al. Intra-ophthalmic artery chemotherapy triggers vascular toxicity through endothelial cell inflammation and leukostasis. Invest Ophthalmol Vis Sci 2012;53:2439-45.

18 Tse BC, Steinle JJ, Johnson D, et al. Superselective intraophthalmic artery chemotherapy in a nonhuman primate model: histopathologic findings. JAMA Ophthalmol 2013;131:903-11.

19 Maidana DE, Pellegrini M, Shields JA, et al. Choroidal thickness after intra-arteria chemotherapy for retinoblastoma. Retina (Philadelphia, Pa) 2014;34:2103-9.

20 Tse BC, Kaste SC, Brennan R, et al. Enophthalmos and choroidal atrophy after intraophthalmic artery chemotherapy for retinoblastoma. Ophthalmology 2015;122:435-7.

21 Bonanomi MT, Saito OC, de Lima PP, et al. Blood flow velocity in monocular retinoblastoma assessed by color Doppler. Clinics (Sao Paulo) 2015;70:797-803. 